MODELING, IDENTIFICATION AND CONTROL, 1998, VOL. 19, NO. 1, 13-29

doi:10.4173/mic.1998.1.2

\title{
Speed and surge control for a low order centrifugal compressor $\operatorname{model} \dagger$
}

\author{
JAN TOMMY GRAVDAHL and OLAV EGELAND
}

Keywords: Centrifugal compressor, modelling, surge control, speed control

A model of a variable speed centrifugal compression system is presented. The model is based on the work of Greitzer (1976), but the compressor characteristic is developed by modelling the losses in the compressor. For surge control, a close coupled valve is employed. This valve is placed immediately downstream of the compressor, and the pressure drop over the valve is used as the control variable. This makes it possible to manipulate the shape of the equivalent compressor, consisting of compressor and valve. The speed of the compressor is controlled with a PI-controller. Semi-global exponential stability of the model with the proposed controllers is proven by the use of Lyapunovs theorem.

\section{Introduction}

Compressor surge is an axisymmetric oscillation of the mass flow and pressure rise in a compression system. Modeling and control of these oscillations is of considerable interest since surge limits the useful range of mass flows where the compressor operate stably. Large amplitude surge can also damage the compressor. A low order lumped parameter model capable of simulating these oscillations was first introduced in Greitzer (1976), where a model for axial compressor mass flow and pressure rise was presented. In K. E. Hansen et al. (1981) it was shown that the model of Greitzer (1976) is also applicable to centrifugal (radial) compressors.

Over the last decade many papers covering the area of surge control have been published. A recent review can be found in de Jager (1995). Of many possible actuation schemes, close coupled valve (CCV) control is considered one of the most promising (Dussourd et al. 1977, Greitzer 1981, Pinsley et al. 1991, Simon et al. 1993, Jungovski et al. 1996, van de Wal et al. 1997). Experimental results of CCV control is reported in Dussourd et al. (1977) and Jungovski et al. (1996). The cause of surge in a compression system is that the throttle line crosses the compressor characteristic in an area of positive compressor characteristic slope. A close coupled valve is placed immediately downstream of the compressor (hence close coupled), and active control of the valve pressure drop is utilized to make the slope of the equivalent compressor (compressor in series with the valve) negative and thereby stabilizing the system.

This approach was used in Jungovski et al. (1996), Pinsley et al. (1991) and Simon et al. (1993) for surge control of the model of Greitzer (1976). Linear stability analysis was used in designing control laws resulting in local stability results. In Simon and Valavani (1991) pressure disturbances were included in the analysis, and a nonlinear

Received 3 October 1997.

†C IEEE. Reprinted, with permission, from Proceedings of the Sixth IEEE Conference on Control Applications, Hartford, Connecticut, October 5-7, pp. 344-349, 1997.

Department of Engineering Cybernetics, Norwegian University of Science and Technology, N-7034 Trondheim, Norway. Email: Tommy.Gravdahl@itk.ntnv.no, Olav.Egeland@itk. ntnv.no 


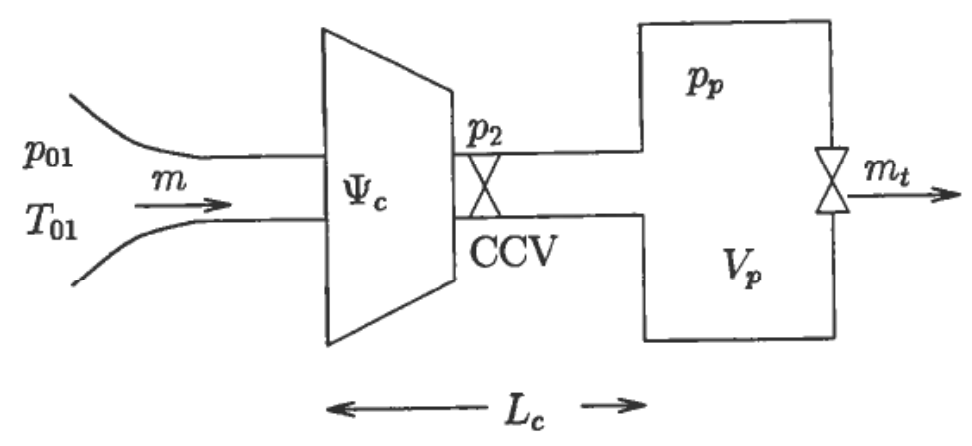

Figure 1. Compression system.

CCV control law was designed for the model of Greitzer (1976) using Lyapunovs method. By applying backstepping, Gravdahl and Egeland (1997b) developed nonlinear surge controllers for the same model, but included disturbances both in mass flow and pressure. Global stability results were presented. In Gravdahl and Egeland (1997d) certain passivity properties of the model was utilized in designing a CCV control law. A CCV can also be used in control of rotating stall in axial compressors, as shown in Gravdahl and Egeland (1997c).

One drawback of CCV control is that the valve introduces a pressure drop in the compression system as discussed in Simon and Valavani (1991). When using the valve as a steady-state device, such as in Dussourd et al. (1977), this loss may become unaccceptably large. However, as pointed out in Pinsley et al. (1991) and Simon and Valavani (1991), a time varying valve will introduce considerably less pressure drop than a valve with constant pressure drop.

Since compressors are variable speed machines, it is of interest to investigate the influence of speed transients on the surge dynamics. Models describing this interaction was developed in Eveker and Nett (1991) for axial compressors and in Fink et al. (1992) for centrifugal compressors. As surge can occur during acceleration of the compressor speed, it is of major concern to develop controllers that simultaneously can control both surge and compressor speed. A model of an axial compression system which both takes variable speed and rotating stall into account is presented in Gravdahl and Egeland (1997a).

In this paper we present a surge controller for a variable speed centrifugal compressor. The speed is controlled with a PI-control law. Inspired by Ferguson (1963) and Watson and Janota (1982), we make a departure from the 3rd order polynomial approximation of the compressor characteristic commonly used in the control literature. Fluid friction and incidence losses in the compressor stage are modeled, and a variable speed compressor characteristic is developed based on this. Semi-global exponential stability results for the proposed controllers are given using Lyapunovs method. The results are confirmed through simulations.

\section{Preliminaries}

We are considering a compression system consisting of a centrifugal compressor, close coupled valve, compressor duct, plenum volume and a throttle. The throttle can be regarded as a simplified model of a turbine. The system is shown in Figure 1. The model to be used for controller design is in the form 


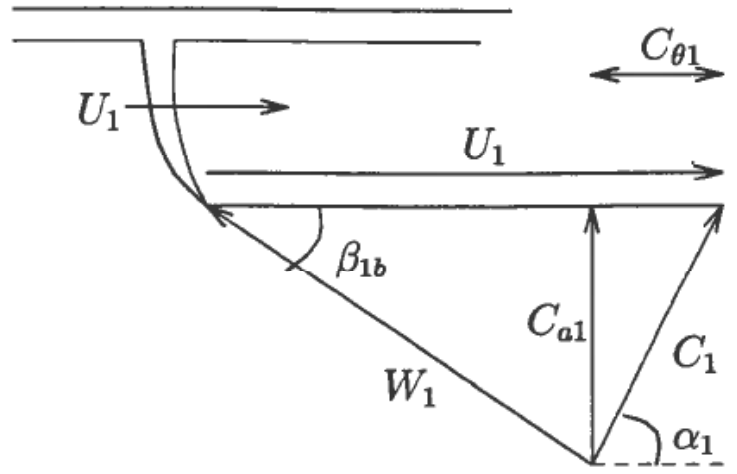

Figure 2. Velocity triangle at inducer.

$$
\begin{aligned}
\dot{p}_{p} & =\frac{a_{01}^{2}}{V_{p}}\left(m-m_{t}\right) \\
\frac{L_{c}}{A_{1}} \dot{m} & =p_{2}(m, \omega)-p_{p} \\
J \dot{\omega} & =\tau_{t}-\tau_{c}(m, \omega),
\end{aligned}
$$

where $m$ is the compressor mass flow, $p_{2}$ is the pressure downstream of the compressor, $a_{01}$ is the inlet stagnation sonic velocity, $L_{c}$ is the length of compressor and duct, $A_{1}$ is the area of the impeller eye (used as reference area), $J$ is the spool moment of inertia, $\tau_{t}$ is the drive torque and $\tau_{c}$ is the compressor torque. The two first equations of (1) are equivalent to the model of Greitzer (1976).

The angular speed of the compressor $\omega$ is included as a state in addition to mass flow and pressure rise which are the states in Greitzer's surge model. The equation for $\dot{p}_{p}$ follows from the mass balance in the plenum, assuming the plenum process isentropic, and the equation for $\dot{m}$ follows from the impulse balance in the duct. In the following, the model (1) will be developed in detail. In particular, expressions must be found for the terms $p_{2}$ and $\tau_{c}$. It will also be shown that an expression for the compressor characteristic results from this derivation.

Incoming gas (air) enters the impeller eye (the inducer) of the compressor with velocity $C_{1}$, see Figure 2 . The mass flow $m$ and velocity $C_{1}$ are given by

$$
C_{1}=\frac{1}{\rho_{01} A_{1}} m
$$

where $\rho_{01}$ is the assumed constant stagnation inlet density. The tangential velocity $U_{1}$ of the inducer is calculated as

$$
U_{1}=\frac{D_{1}}{2} \omega=D_{1} \pi N,
$$

where $\omega$ is the angular velocity of the impeller and $N$ is the number of revolutions per second. The average diameter $D_{1}$ is defined according to

$$
D_{1}^{2}=\frac{1}{2}\left(D_{t 1}^{2}+D_{h_{1}}^{2}\right)
$$

where $D_{t 1}$ and $D_{h_{1}}$ are the diameters at inducer tip and hub casing respectively. The circle with diameter $D_{1}$ and area $A_{1}$ divides the inducer in two annuli of equal area. The 


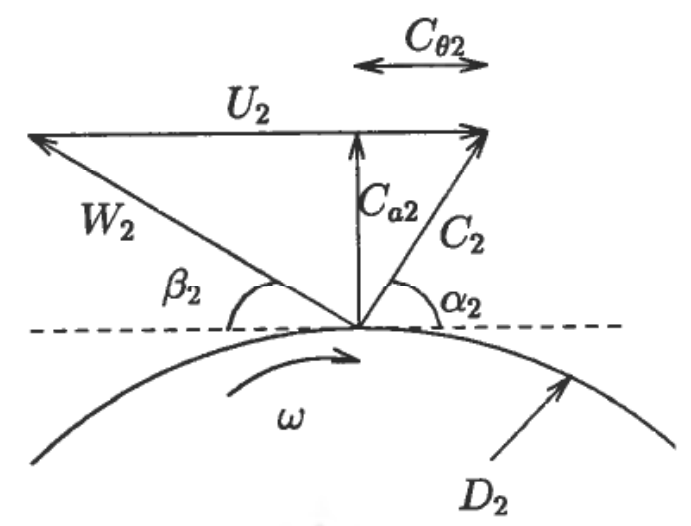

Figure 3. Velocity triangle at impeller tip.

compressed gas leaves the impeller at the impeller tip with velocity $C_{2}$ as shown in Figure 2. The diameter at the impeller tip is $D_{2}$ and the tangential tip velocity is $U_{2}$.

\section{Energy transfer and compressor torque}

\subsection{Ideal case}

For a compressor, applied torque equals the change in angular momentum of the fluid

$$
\tau_{c}=m\left(r_{2} C_{\theta 2}-r_{1} C_{\theta 1}\right),
$$

where $\tau_{c}$ is the compressor torque, $r_{1}=\frac{D_{1}}{2}, r_{2}=\frac{D_{2}}{2}$ and $C_{\theta 2}$ is the tangential component of the gas velocity $C_{2}$. Power delivered to the fluid is

$$
\begin{aligned}
\dot{W}_{c} & =\omega \tau_{c}=\omega m\left(r_{2} C_{\theta 2}-r_{1} C_{\theta 1}\right) \\
& =m\left(U_{2} C_{\theta 2}-U_{1} C_{\theta 1}\right)=m \Delta h_{0 c, \text { ideal }}
\end{aligned}
$$

where $\Delta h_{0 c \text {,ideal }}$ is the specific enthalpy delivered to the fluid without taking account for losses. Equation (6) is known as Eulers pump equation. For simplicity the following two assumptions are made. A radially vaned (no backsweep) impeller is considered with $\beta_{2 b}=90^{\circ}$, and there is no pre-whirl, that is $\alpha_{1}=90^{\circ} \Rightarrow C_{\theta 1}=0$. The slip factor is defined as

$$
\sigma \triangleq \frac{C_{\theta 2}}{U_{2}} \approx 1-\frac{2}{i} .
$$

The approximation is known as Stanitz' formula where $i$ is the number of compressor blades. From (6), and using (7), we have that the ideal specific enthalpy delivered to the fluid is

$$
\Delta h_{0 c, \text { ideal }}=\frac{\dot{W}_{c, \text { ideal }}}{m}=\sigma U_{2}^{2},
$$

and that the compressor torque is

$$
\tau_{c}=m r_{2} C_{\theta 2}=m r_{2} \sigma U_{2} .
$$




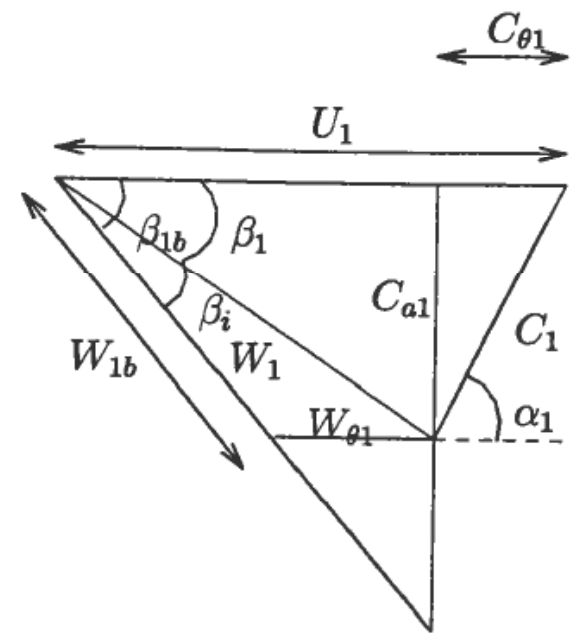

Figure 4. Incidence angles.

Notice that $\Delta h_{0 c, \text { ideal }}$ is independent of mass flow $m$, and ideally we would have the same energy transfer for all mass flows. ${ }^{1}$ However, due to various losses, the energy transfer is not constant, and we now include this in the analysis. According to Watson and Janota (1982), the two major losses, expressed as specific enthalpies, are:

1. Incidence losses, $\Delta h_{i}$

2. Fluid friction losses, $\Delta h_{f}$.

Other losses such as inlet casing losses, disc friction losses, leakage losses, diffuser losses, and collector losses do occur, but these will not be considered here. Moreover, the incidence losses and fluid friction losses play an important role in determining the region of stable operation for the compressor. Depending on the design of the diffuser, both incidence losses and friction losses may take place in this part of the compressor. Such losses can easily be included in the model, but are ignored for simplicity.

\subsection{Incidence losses}

The velocity of the incoming gas relative to the inducer is denoted $W_{1}$. In off-design operation there will be a mismatch between the fixed blade angle $\beta_{1 b}$ and the direction of the gas stream $\beta_{1}=\beta_{1}\left(U_{1}, C_{1}\right)$, as shown in Figure $2 \mathrm{~d}$. The angle of incidence is defined by

$$
\beta_{i} \triangleq \beta_{1 b}-\beta_{1} .
$$

As the gas hits the inducer, its velocity instantaneously changes its direction to comply with the blade inlet angle $\beta_{1 b}$. The direction is changed from $\beta_{1}$ to $\beta_{1 b}$, and the energy associated with the tangential component $W_{\theta 1}$ of the velocity is lost. That is, the incidence loss can be expressed as

$$
\Delta h_{i}=\frac{W_{\theta 1}^{2}}{2} .
$$

\footnotetext{
${ }^{1}$ If backswept impeller blades, $\beta_{2 b}<90^{\circ}$, were considered, $\Delta h_{0 c, \text { ideal }}$ would decrease with increasing $m$.
} 
From Figure $2 d$ it is easily seen that

Furthermore

$$
\cos \beta_{1}=\frac{U_{1}-C_{\theta 1}}{W_{1}} \text { and } \sin \beta_{1}=\frac{C_{a 1}}{W_{1}} .
$$

$$
W_{\theta 1}=\frac{\sin \left(\beta_{1 b}-\beta_{1}\right)}{\sin \beta_{1 b}} W_{1}=\left(\cos \beta_{1}-\cot \beta_{1 b} \sin \beta_{1}\right) W_{1} .
$$

Inserting (12) in (13) gives

$$
W_{\theta 1}=U_{1}-C_{\theta 1}-\cot \beta_{1 b} C_{a 1} .
$$

and the incidence loss (11) can be written

$$
\Delta h_{i}=\frac{1}{2}\left(U_{1}-C_{\theta 1}-\cot \beta_{1 b} C_{a 1}\right)^{2}=\frac{1}{2}\left(U_{1}-\frac{\cot \beta_{1 b} m}{\rho_{01} A_{1}}\right)^{2}
$$

where the second equality is found using (2). Similar results are presented in chap. 5 in Ferguson (1963).

\subsection{Frictional losses}

According to Ferguson (1963) loss due to friction can be calculated as

$$
\Delta h_{f}=C_{h} \frac{l}{D} \frac{W_{1 b}^{2}}{2},
$$

where $C_{h}$ is the surface friction loss coefficient, $l$ is the mean channel length and $D$ is the mean hydraulic channel diameter. This friction loss is actually calculated for constant area pipes of circular cross section. The friction loss coefficient $C_{h}$ is defined as (Watson and Janota 1982)

$$
C_{h}=4 f,
$$

where the friction factor $f$ depends on the Reynolds number. Many different formulas for the friction factor have been published, see e.g., Ferguson (1963) or White (1986). Here we will use Blasius' formula

$$
f=0.3164(R e)^{-0.25},
$$

which was found empirically for turbulent flow in smooth pipes with Reynolds number $\operatorname{Re}$ below 100,000 (White 1986). The mean hydraulic channel diameter $D$ is defined as

$$
D=\frac{4 A}{a},
$$

where the cross section area $A$ and perimeter $a$ are mean values for the passage. The mean hydraulic diameter $D$ corresponds to a circle with area $A$ and perimeter $a$. Although the passage between the blades in the compressor are neither circular nor of constant area, Baljé (1952) reports of good agreement between theory and measurement using (18).

Using Figure 2d, it is seen that

and using $\sin \beta_{1}=\frac{C_{a l}}{W_{1}}$ we get

$$
\frac{W_{1 b}}{\sin \beta_{1}}=\frac{W_{1}}{\sin \beta_{1 b}}
$$

$$
W_{1 b}=\frac{C_{1}}{\sin \beta_{1 b}} \text {. }
$$


Inserting (2) and (21) in (16) gives

$$
\Delta h_{f}=\frac{C_{h} l}{2 D \rho_{1}^{2} A_{1}^{2} \sin ^{2} \beta_{1 b}} m^{2}=k_{f} m^{2} .
$$

As can be seen the friction losses are quadratic in mass flow and independent of wheel speed $U$. Equation (22) represents the loss due to friction of a mass flow $m$ through a pipe of hydraulic diameter $D$.

\section{Energy transfer and pressure rise}

Including the losses, the total specific energy transfer can be calculated by subtracting (22) and (15) from (8):

$$
\Delta h_{0 c}\left(U_{1}, m\right)=\Delta h_{0 c, \text { ideal }}-\Delta h_{f}-\Delta h_{i} .
$$

$\Delta h_{0 c}$ is a 2 nd degree polynomial in $m$, and as opposed to the ideal case, we see that energy transfer to the fluid is varying with mass flow $m$. This is shown in Figure 5 .

To find an expression for the pressure rise we now need a relation between pressure rise and energy transfer. The pressure rise is modeled as

$$
p_{2}=\left(1+\frac{2_{\mathrm{i}}\left(U_{1}, m\right) \Delta h_{0 c, \text { ideal }}}{T_{01} c_{p}}\right)^{\frac{\gamma}{\gamma-1}}=\Psi_{c}\left(U_{1}, m\right) p_{01},
$$
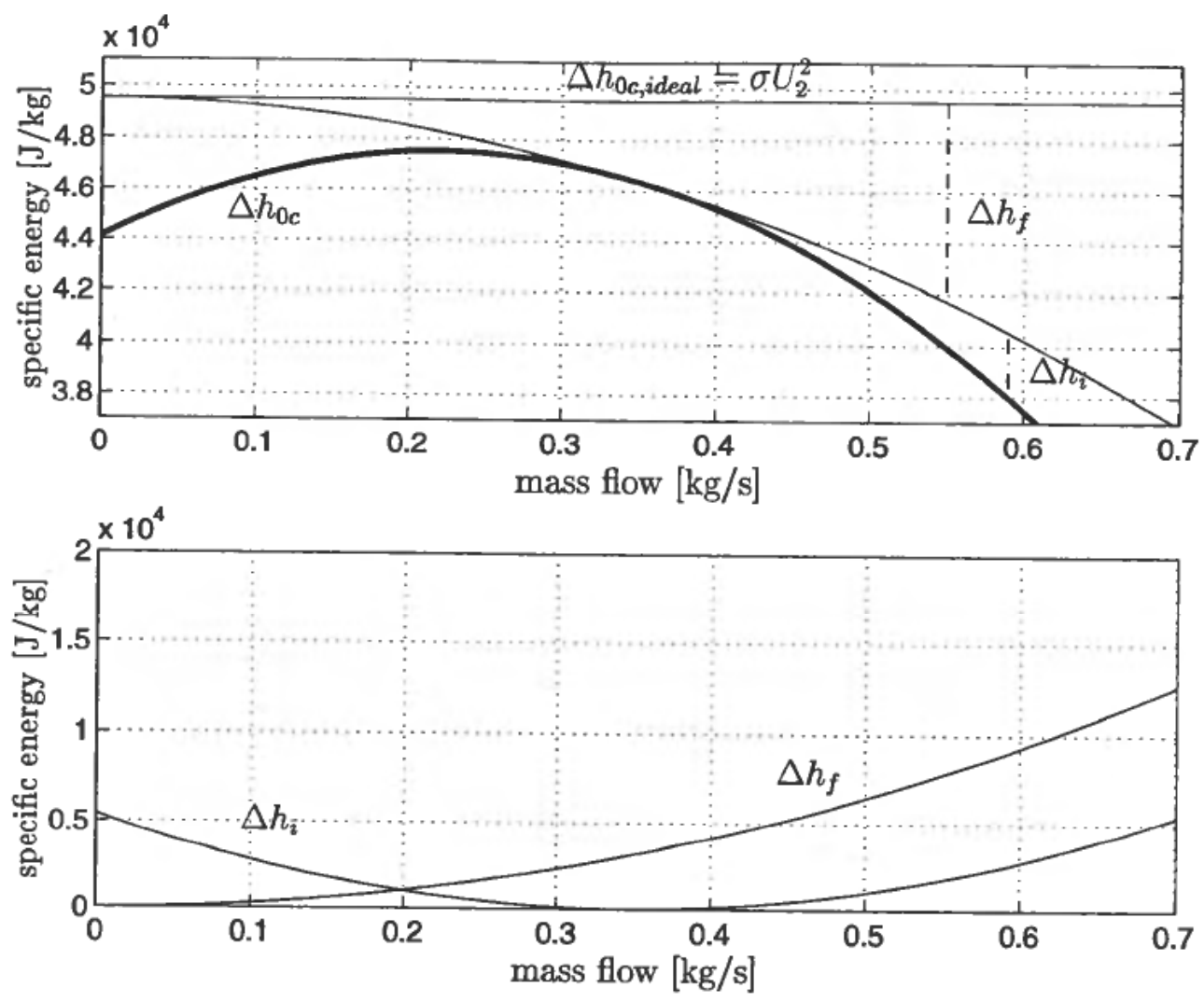

Figure 5. Energy transfer for $N=35,000 \mathrm{rpm}$. 
where the losses have been taken into account, and $\Psi_{c}\left(U_{1}, m\right)$ is the compressor characteristic. We now have an expression for the pressure $p_{2}$ needed in the model (1). The inlet stagnation temperature $T_{01}$, specific heat capacity $c_{p}$, and $\gamma$ are assumed constant. The isentropic efficiency $\eta_{i}$ of the compressor is defined as (Cumpsty 1989)

$$
\eta_{i}\left(U_{1}, m\right)=\frac{\Delta h_{0 c, \text { ideal }}}{\Delta h_{0 c, \text { ideal }}+\Delta h_{\mathrm{t}}+\Delta h_{\mathrm{i}}} .
$$

The ideal energy transfer and the losses are shown in Figure 5. The curves are calculated for a compressor speed of $N=35,000 \mathrm{rpm}$. The compressor pressure characteristic as calculated from equation (24) is shown in Figure 6.

In Figures 5 and 6 the numerical values for the compressor parameters is taken from Fink et al. (1992). Comparing the compressor map in Figure 6 with Figure 3 in Fink et al. (1992), which is based on physical measurements, we see that they are almost similar.

The surge line is the line in the compressor map that divides the map into an area of stable compressor operation and unstable (surge) operation. The line passes through the local maxima of the constant speed lines in the map, and is drawn with a solid line in Figure 6.

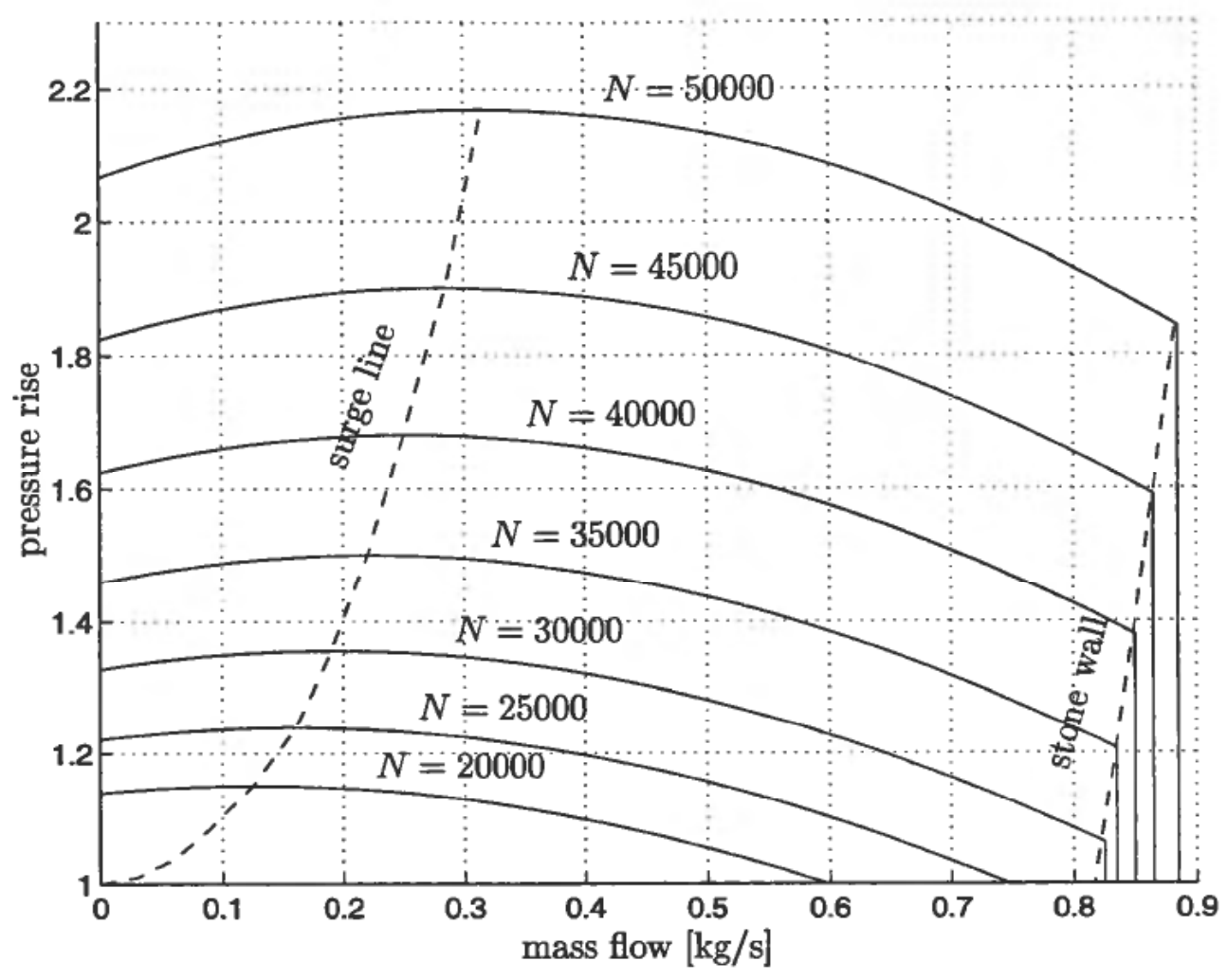

Figure 6. Centrifugal compressor characteristic. 


\section{Choking}

When the flow reaches the speed of sound at some cross-section of the compression system, the flow chokes. For now it is assumed that the flow chokes in the impeller. Assuming isentropic flow, Dixon (1978) calculated the choking flow as

where

$$
m_{\text {choke }}\left(U_{1}\right)=A_{1} \rho_{01} a_{01}\left[\frac{2+(\gamma-1)\left(\frac{U_{1}}{a_{01}}\right)^{2}}{\gamma+1}\right]^{\frac{(\gamma+1)}{2(\gamma-1)}},
$$

$$
\rho_{01}=\frac{p_{01}}{R T_{01}} \text { and } a_{01}=\sqrt{\gamma R T_{01}}
$$

is the inlet stagnation density and inlet stagnation sonic velocity, respectively. It is seen that the choking mass flow is dependent on blade speed $U_{1}$. Thus the impeller can accept a greater limiting mass flow rate at higher rotational speeds. The effect of choking can be seen in Figure 6, where a choke line, also known as a stone wall, has been drawn. In this paper, the effect of choking is treated in an approximate manner. Due to sonic effects, the pressure rise would fall off more gradually when approaching the stone wall than shown in Figure 6.

\section{Dynamic model}

To complete the dynamic model (1), an expression for the throttle mass flow is needed. The mass flow $m_{t}$ through the throttle is modeled as

$$
m_{t}=k_{t} \sqrt{p_{p}-p_{01}},
$$

where $k_{t}$ is the throttle gain proportional to throttle opening and $p_{p}$ is the plenum pressure. The momentum balance of the spool is

Using (3) it is seen that

$$
J \dot{\omega}=\tau_{t}-\tau_{c} .
$$

$$
\omega=\frac{2 U_{1}}{D_{1}} \Rightarrow \dot{\omega}=\frac{2 \dot{U}_{1}}{D_{1}},
$$

and thus we get a differential equation for $U_{1}$,

$$
\dot{U}_{1}=\frac{D_{1}}{2 J}\left(\tau_{t}-\tau_{c}\right) \text {. }
$$

The drive torque $\tau_{t}$ may be delivered by a turbine, and will be used as a control variable for speed control. The compressor and spool can only rotate in one direction, and the speed is assumed upper bounded:

$$
0 \leq U_{1}(t)<U_{m} .
$$

Using (9) and (31), and inserting equations (24) and (28) in (1), we get the following dynamic model for the compression system ${ }^{2}$

\footnotetext{
${ }^{2}$ It is worth noticing that a time varying $U$ is equivalent with a time varying B-parameter (Fink et al. 1992). Greitzer's B-parameter as defined in Greitzer (1976) is given by

$$
B=\frac{U_{1}}{2 a_{01}} \sqrt{\frac{V_{p}}{A_{1} L_{c}}},
$$

where $V_{p}$ is the plenum volume and $L_{c}$ is the length of the compressor and duct. Using (31), a nonlinear differential equation for $B$ can be found.
} 


$$
\begin{aligned}
\dot{p}_{p} & =\frac{a_{01}^{2}}{V_{p}}\left(m-k_{t} \sqrt{p_{p}-p_{01}}\right) \\
\dot{m} & =\frac{A}{L}\left(\left(1+\frac{\eta_{i}\left(U_{1}, m\right) \Delta h_{0 c, \text { ideat }}}{T_{01} c_{p}}\right)^{\frac{\gamma}{\gamma-1}} p_{01}-p_{p}\right) \\
\dot{U}_{1} & =\frac{D_{1}}{2 J}\left(\tau_{t}-\tau_{c}\left(U_{1}, m\right)\right) .
\end{aligned}
$$

\section{Surge control idea}

The reason for equilibria to the left of the surge line being unstable, and causing the compressor to go into surge, is the positive slope of the characteristic in this area. From Figure 5 it is seen that the positive slope is due to the incidence losses at low mass flows. From the expression for the incidence loss, equation (15), it is clear that a variable blade angle $\beta_{1 b}$ would make it possible to minimize the incidence losses over a range of mass flows. Thus variable inducer blades might be used as a means of surge stabilization.

On the other hand, the maximum energy transfer and minimum incidence loss do not occur for the same mass flow. This is due to the friction losses. The friction shifts the point of maximum energy transfer, and consequently pressure rise, to the left of the point of minimum incidence loss. From this we conclude that the friction losses in fact have a stabilizing effect, and introducing additional fluid friction would move the point of maximum energy transfer to the left. The effect of this, when considering the compressor map in Figure 8 is that the surge line will be shifted to the left, and the area of stable compressor operation is expanded.

This motivates us to introduce a valve in series with compressor. The pressure drop over this valve will serve as the control variable, and it will be used to introduce additional friction at low mass flows in order to avoid surge. The use of a close coupled valve for constant speed centrifugal compressor surge control was studied in Dussourd et al. (1977), Simon and Valavani (1991), Pinsley et al. (1991), Jungovski et al. (1996) and van de Wal et al. (1997).

\section{Controller design and stability analysis}

The equivalent compressor characteristic for compressor and close coupled valve is defined as

$$
\Psi_{e}\left(m, U_{1}\right)=\Psi_{c}\left(m, U_{1}\right)-\Psi_{v}(m),
$$

where $\Psi_{v}(m) p_{01}$ is the pressure drop across the CCV and

$$
\Psi_{c}\left(m, U_{1}\right)=\left(1+\frac{\eta_{i}\left(U_{1}, m\right) \Delta h_{0 c, \text { ideal }}}{T_{01} c_{p}}\right)^{\frac{\gamma}{\gamma-1}} .
$$

Assume $p_{0}, m_{0}$ to be the equilibrium values of pressure and mass flow as dictated by the intersection of the throttle and compressor characteristics, and $U_{d}$ to be the desired spool speed. Define the following error variables

$$
\hat{p}=p-p_{0}, \hat{m}=m-m_{0}, \hat{U}=U_{1}-U_{d} .
$$

The equations of motion (33) are now transformed so that the origin becomes the equilibrium under study. Notice that no assumptions are made about the numeric values 
of $m_{0}$ and $p_{0}$, so that the equilibrium can be on either side of the surge line. By including the CCV (34), the model (33) can be written in the form

$$
\begin{aligned}
\hat{\hat{p}} & =\frac{a^{2}}{V_{p}}\left(\hat{m}-\hat{m}_{t}(\hat{p})\right) \\
\hat{\hat{m}} & =\frac{A}{L}\left(\left(\hat{\Psi}_{c}(\hat{m}, \hat{U})-\hat{\Psi}_{v}(\hat{m})\right) p_{01}-\hat{p}\right) \\
\hat{U}_{1} & =\frac{D_{1}}{2 J}\left(\hat{\tau}_{t}-\hat{\tau}_{c}\right)
\end{aligned}
$$

where a hat denotes transformation to the new coordinates $(36)$, and $(\hat{p} \hat{m} \hat{U})^{\mathrm{T}}=(000)^{\mathrm{T}}$ is the equilibrium.

Theorem 1 The surge control law

and the speed control law

$$
\hat{\Psi}_{v}=k_{\nu} \hat{m},
$$

where

$$
\begin{aligned}
& \hat{\tau}_{t}=-k_{p} \hat{U}-k_{i} \hat{I}, \\
& \dot{\hat{I}}=\hat{U},
\end{aligned}
$$

$$
k_{p}>0, k_{i}>0 \text { and } k_{v}>\sup _{\hat{U}, \hat{m}}\left\{\frac{\partial \hat{\Psi}_{c}(\hat{m}, \hat{U})}{\partial \hat{m}}\right\}+\delta_{1}
$$

makes the origin of (37) semi-global exponentially stable. That is, desired compressor speed $U_{d}$ is achieved regardless of the equilibrium being to the right or to the left of the original surge line. The design parameter $\delta_{1}>0$ in (40) can be chosen freely. The integral term in (39) is added in order to robustify the controller wrt to unmodeled torques, arising from disc friction, leakages, etc.

\section{Proof 1 Define}

$$
\boldsymbol{z} \triangleq\left(\begin{array}{c}
\hat{U} \\
\hat{I}
\end{array}\right) \text { and } \boldsymbol{P} \triangleq\left(\begin{array}{cc}
\frac{2 J}{D_{1}} & \mu \\
\mu & k_{i}
\end{array}\right)
$$

where $\mu>0$ and $k_{i}>0$ are design parameters. Consider the following Lyapunov function candidate

where

$$
V(\hat{p}, \hat{m}, \hat{U}, \hat{I})=\frac{1}{2}\left(V_{\hat{p}}+V_{\hat{m}}+V_{\text {spool }}\right),
$$

$$
V_{\hat{p}}=\frac{V_{p}}{a_{01}^{2} \rho_{01}} \hat{p}^{2}, V_{\hat{m}}=\frac{L}{A_{1} \rho_{01}} \hat{m}^{2} \text { and } V_{\text {spool }}=\boldsymbol{z}^{\mathrm{T}} \mathbf{P} z
$$

As all coefficients in (42) are constant it follows that $V$ is positive definite and radially unbounded, provided that $\mu$ is chosen such that $\boldsymbol{P}>0$, that is

$$
\mu<\sqrt{\frac{2 J k_{i}}{D_{1}}} .
$$

Calculating the time derivative of (42) along the solutions of (37) and accounting for (39) gives

$$
\begin{aligned}
\dot{V}= & \hat{m}\left(\hat{\Psi}_{c}(\hat{m}, \hat{U})-\hat{\Psi}_{v}(\hat{m})\right) \frac{p_{01}}{\rho_{01}}-\frac{1}{\rho_{01}} \hat{p} \hat{m}_{t}(\hat{p})-k_{p} \hat{U}^{2} \\
& +\mu \hat{U}^{2}-\frac{\mu k_{i} D_{1}}{2 J} \hat{I}^{2}-\frac{\mu k_{p} D_{1}}{2 J} \hat{U} \hat{I}-\hat{U} \hat{\tau}_{c}-\frac{\mu D_{1}}{2 J} \hat{I} \hat{\tau}_{c} .
\end{aligned}
$$


The last term in (45) can be upper bounded as

$$
-\frac{\mu D_{1}}{2 J} \hat{I} \hat{\tau}_{c} \leq \frac{\mu D_{1}}{2 J}\left(\frac{\sigma D_{2}^{2} U_{m}}{2 D_{1}}\left(\frac{\hat{m}^{2}}{\eta_{1}}+\eta_{1} \hat{I}^{2}\right)+m_{0} \hat{U} \hat{I}\right)
$$

using (9), (32) and Young's inequality. The parameter $\eta_{1}>0$ can be chosen freely. The $\hat{U} \hat{\tau}_{c}$-term can be upper bounded as

$$
-\hat{U} \hat{\tau}_{c}<-\frac{D_{2}^{2} \sigma}{2 D_{1}}|m| \hat{U}^{2}+\frac{D_{2}^{2} \sigma}{4 D_{1}}\left(\frac{\hat{m}^{2}}{\eta_{2}}+\eta_{2}\left(U_{d} \hat{U}\right)^{2}\right) .
$$

Now, (45) can be upper bounded as

$$
\begin{gathered}
\dot{V} \leq \hat{m}\left(\hat{\Psi}_{c}(\hat{m}, \hat{U})-\hat{\Psi}_{v}(\hat{m})\right) \frac{p_{01}}{\rho_{01}}-\frac{D_{2}^{2} \sigma}{2 D_{2}}|m| \hat{U}^{2} \\
+\left(\frac{\sigma \mu D_{2}^{2} U_{m}}{8 J \eta_{1}}+\frac{D_{2}^{2} \sigma}{4 D_{1} \eta_{2}}\right) \hat{m}^{2}-\frac{1}{\rho_{01}} \hat{p} \hat{m}_{t}(\hat{p})-z^{\mathrm{T}} \boldsymbol{R} z,
\end{gathered}
$$

where

$$
\boldsymbol{R}=\left(\begin{array}{cc}
k_{p}-\mu-\frac{D_{2}^{2} \sigma U_{d}^{2} \eta_{2}}{4 D_{1}} & \frac{\mu}{4 J}\left(D_{1} k_{p}-\frac{\sigma D_{2}^{2} m_{0}}{4}\right) \\
\frac{\mu}{4 J}\left(D_{1} k_{p}-\frac{\sigma D_{2}^{2} m_{0}}{4}\right) & \frac{\mu}{2 J}\left(k_{i} D_{1}-\frac{\sigma D_{2}^{2} U_{m} \eta_{1}}{4}\right)
\end{array}\right) .
$$

Demanding $\boldsymbol{R}>0$ gives the following conditions on $k_{p}, \eta_{1}$ and $\mu$

$$
\begin{gathered}
k_{p}>\frac{D_{2}^{2} \sigma U_{d}^{2} \eta_{2}}{4 D_{1}}, \\
\eta_{1}<\frac{4 k_{i} D_{1}}{\sigma D_{2} U_{m}} \text { and } \\
\mu<\min \left\{k_{p}-\frac{D_{2}^{2} \sigma U_{d}^{2} \eta_{2}}{4 D_{1}}, \frac{\left(k_{p}-\frac{D_{2}^{2} \sigma U_{d}^{2} \eta_{2}}{4 D_{1}}\right)\left(k_{i} D_{1}-\frac{\sigma D_{2} U_{m} \eta_{4}}{4}\right)}{k_{i} D_{1}-\frac{\sigma D_{2} U_{m} \eta_{4}}{4}+\frac{1}{8 J}\left(D_{1} k_{p}-\frac{\sigma D_{2} m_{0}}{4}\right)^{2}}\right)
\end{gathered}
$$

It is assumed that $\hat{m}_{t}$ satisfies the sector condition

$$
\hat{p} \hat{m}_{t}(\hat{p})>\delta_{2} \hat{p}^{2},
$$

that is, the throttle is assumed passive as in Simon and Valavani (1991). As $\hat{p} \hat{m}_{t}(\hat{p})$ is of order $\frac{3}{2}$ in $\hat{p},(53)$ does not hold globally. However for a given $\hat{p}_{\max }$ such that

$$
|\hat{p}(t)| \leq p_{m} \forall t>0
$$

it will always be possible to choose $\delta_{2}$ small enough for (53) to hold for $|\hat{p}(t)| \leq \hat{p}_{\max }$. Now, the CCV pressure drop $\Psi_{v}(\hat{m})$ is to be chosen such that for the first term in (48), the condition

$$
-\hat{m}\left(\hat{\Psi}_{c}(\hat{m}, \hat{U})-\hat{\Psi}_{v}(\hat{m})\right) \frac{p_{01}}{\rho_{01}}>0 \forall \hat{U}
$$

is satisfied. Since $\frac{p_{01}}{\rho_{01}}>0$, sufficient conditions for (55) to hold is

$$
\begin{gathered}
-\left.\left(\hat{\Psi}_{c}(\hat{m}, \hat{U})-\hat{\Psi}_{v}(\hat{m})\right)\right|_{\hat{m}=0}=0 \text { and } \\
\frac{\partial}{\partial \hat{m}}\left(-\hat{\Psi}_{c}(\hat{m}, \hat{U})+\hat{\Psi}_{v}(\hat{m})\right)>0 .
\end{gathered}
$$


It can be recognized that

$$
\begin{aligned}
\hat{\Psi}_{c}(0, \hat{U}) & =\Psi_{c}\left(m_{0}, U_{d}\right)-\Psi_{c}\left(m_{0}, U_{d}\right)=0, \\
\hat{\Psi}_{v}(\hat{m}) & =k_{v} \hat{m} \Rightarrow \hat{\Psi}_{v}(0)=0,
\end{aligned}
$$

and thus (56) is satisfied. From (57), we get

$$
-\frac{\partial}{\partial \hat{m}} \hat{\Psi}_{c}(\hat{m}, \hat{U})+k_{v}>0,
$$

and it follows that choosing $k_{v}$ according to

$$
k_{v}>\sup _{\hat{U}, \hat{m}}\left\{\frac{\partial \hat{\Psi}_{c}(\hat{m}, \hat{U})}{\partial \hat{m}}\right\},
$$

guarantees that (57), and thereby (55) being satisfied. Moreover, if chose

$$
k_{v}>\sup _{\hat{U}, \hat{m}}\left\{\frac{\partial \hat{\Psi}_{c}(\hat{m}, \hat{U})}{\partial \hat{m}}\right\}+\delta_{1},
$$

where $\delta_{1}>0$, we get $\hat{m} \Psi_{v}(\hat{m})>\delta_{1} \hat{m}^{2}$, and (55) is modified to

$$
-\hat{m}\left(\hat{\Psi}_{c}(\hat{m}, \hat{U})-\hat{\Psi}_{v}(\hat{m})\right) \frac{p_{01}}{\rho_{01}}>\frac{p_{01}}{\rho_{01}} \delta_{1} \hat{m}^{2} \forall \hat{U} .
$$

Consequently $\dot{V}$ can now be upper bounded as

$$
\dot{V} \leq-\left(\frac{p_{01}}{\rho_{01}} \delta_{1}-\frac{\sigma \mu D_{2}^{2} U_{m}}{8 J \eta_{1}}-\frac{D_{2}^{2} \sigma}{4 D_{1} \eta_{2}}\right) \hat{m}^{2}-\delta_{2} \hat{p}^{2}-\frac{1}{2} z^{\mathrm{T}} R z \forall \hat{m}, \hat{p}, z
$$

We now set out to compare the coefficients of $V$ and $\dot{V}$. The cross terms in $\hat{U}$ and $\hat{I}$ are upper bounded using Young's inequality,

$$
\begin{gathered}
\mu \hat{U} \hat{I} \leq \frac{\mu}{2}\left(\frac{\hat{U}^{2}}{\eta_{3}}+\eta_{3} \hat{I}^{2}\right) \\
-\frac{\mu\left(4 D_{1} k_{p}-\sigma D_{2}^{2} m_{0}\right)}{2 J} \hat{U} \hat{I} \leq \frac{\mu\left(4 D_{1} k_{p}-\sigma D_{2}^{2} m_{0}\right)}{4 J}\left(\frac{\hat{U}^{2}}{\eta_{4}}+\eta_{4} \hat{I}^{2}\right),
\end{gathered}
$$

where $\eta_{3}>0$ and $\eta_{4}>0$ are constants that can be chosen freely. Using (65) and (66) and comparing the coefficients in (42) and (64), it can be recognized that if the following inequalities are satisfied for some $\alpha>0$

$$
\begin{gathered}
\delta_{1}-\frac{\sigma \mu D_{2}^{2} U_{m} \rho_{01}}{8 J \eta_{1} p_{01}}-\frac{D_{2}^{2} \sigma \rho_{01}}{4 D_{1} \eta_{2} p_{01}}>\alpha \frac{L}{A \rho_{01}} \\
\delta_{2}>\alpha \frac{V_{p}}{a_{01}^{2} \rho_{01}}, \\
k_{p}-\mu-\frac{\mu\left(4 D_{1} k_{p}-\sigma D_{2}^{2} m_{0}\right)}{4 J \eta_{4}}>\propto\left(\frac{J}{D_{1}}+\frac{\mu}{2 \eta_{3}}\right), \\
\mu k_{i}-\frac{\mu\left(4 D_{1} k_{p}-\sigma D_{2}^{2} m_{0}\right)}{4 J} \eta_{4}>\alpha\left(\frac{k_{i}}{D_{1}}+\frac{\alpha \eta_{3}}{2}\right),
\end{gathered}
$$

the following holds

$$
\dot{V} \leq-\alpha V \Rightarrow V(t) \leq V(0) e^{-\alpha t} .
$$

If $\eta_{1}$ is chosen according to (51), and $\delta_{1}$ is chosen according to 

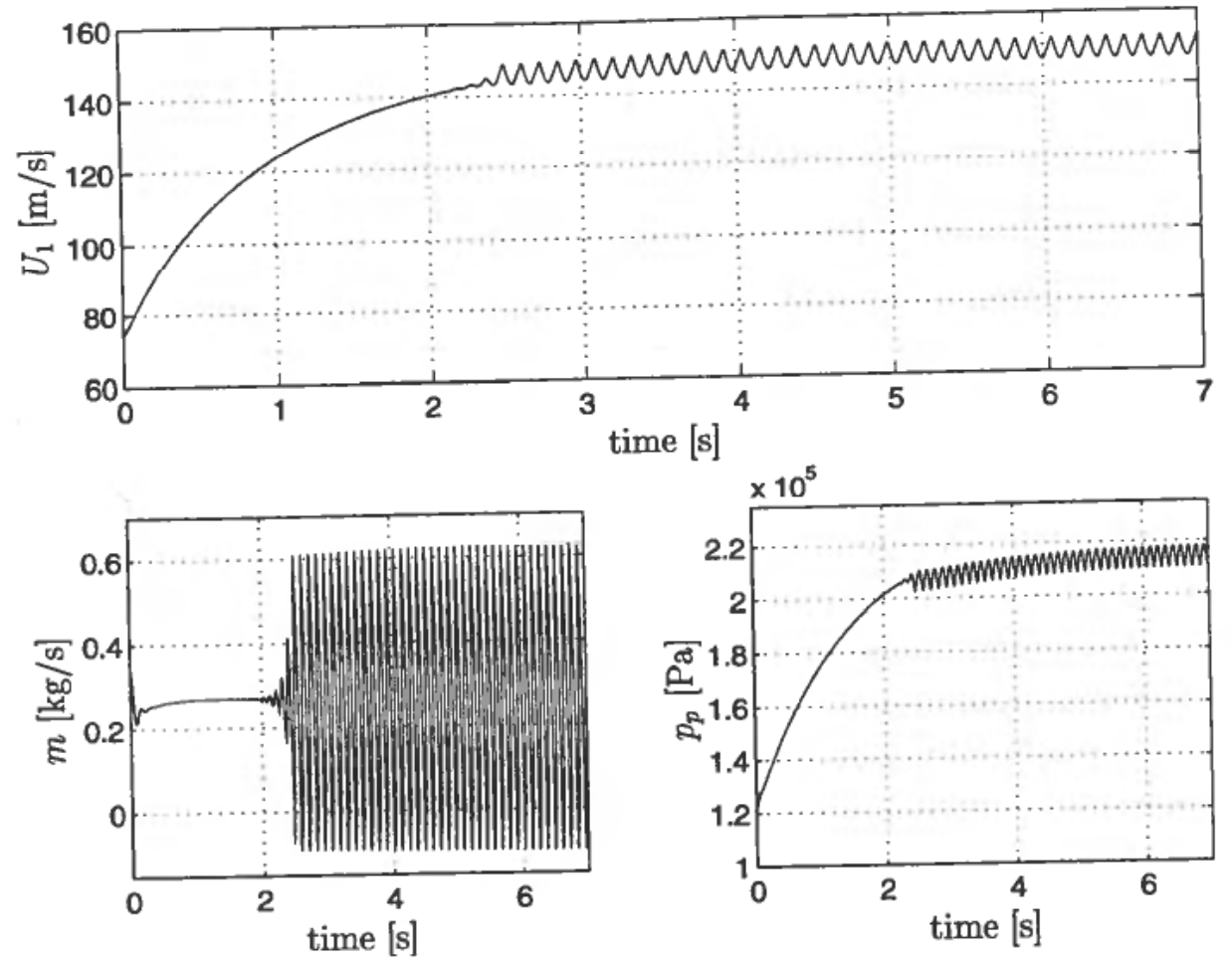

Figure 7. Unstable equilibrium. After $t \approx 2 \mathrm{~s}$ the $(m(t), p(t))$-trajectory crosses the surge line, resulting in deep surge.

$$
\delta_{1}>\frac{\sigma \mu D_{2}^{2} U_{m} \rho_{01}}{8 J \eta_{1} p_{01}}+\frac{D_{2}^{2} \sigma \rho_{01}}{4 D_{1} \eta_{2} p_{01}},
$$

and $k_{v}$ is chosen so that $k_{v}>\sup _{\hat{U}, \hat{m}}\left\{\frac{\partial \hat{\psi}_{c}(\hat{m}, \hat{U})}{\partial \hat{m}}\right\}+\delta_{1}$, and $\mu$ is chosen according to (44) and (52), then the inequalities (67)-(70) are satisfied for some $\alpha>0$. By (71) the origin of (37) is exponentially stable. Due to assumption (54), the stability result holds whenever $|\hat{p}(0)| \leq p_{m}$, and thus the origin is semi-global exponential stable.

Notice that the parameter $\alpha$ can be used to calculate a lower bound on the convergence rate of the system.

\section{Simulations}

In order to model the compressor pressure rise for negative mass flow (deep surge) the following assumption is made:

$$
\Delta h_{0 c}\left(U_{1}, m\right)=\Delta h_{0 c}\left(U_{1},-m\right),
$$

the energy transfer is symmetric in $m$. This assumption is not based on any physical considerations, but the resulting characteristic bear resemblance to real compressor characteristic, e.g. the one in Fink et al. (1992). In Figure 7 the response of the system during surge is showed. The set point for compressor speed was $U_{1 d}=150 \mathrm{~m} /$ $\mathrm{s} \Rightarrow N \approx 50,000 \mathrm{rpm}$ and the speed control law (39) parameters were set to $k_{p}=k_{i}=0.07$. The throttle gain was set to $k_{t}=0.0075$ which gives an unstable equilibrium to the left of the surge line. Notice the oscillations in the compressor speed. These variations in speed during surge were first described in Eveker and Nett (1991). 


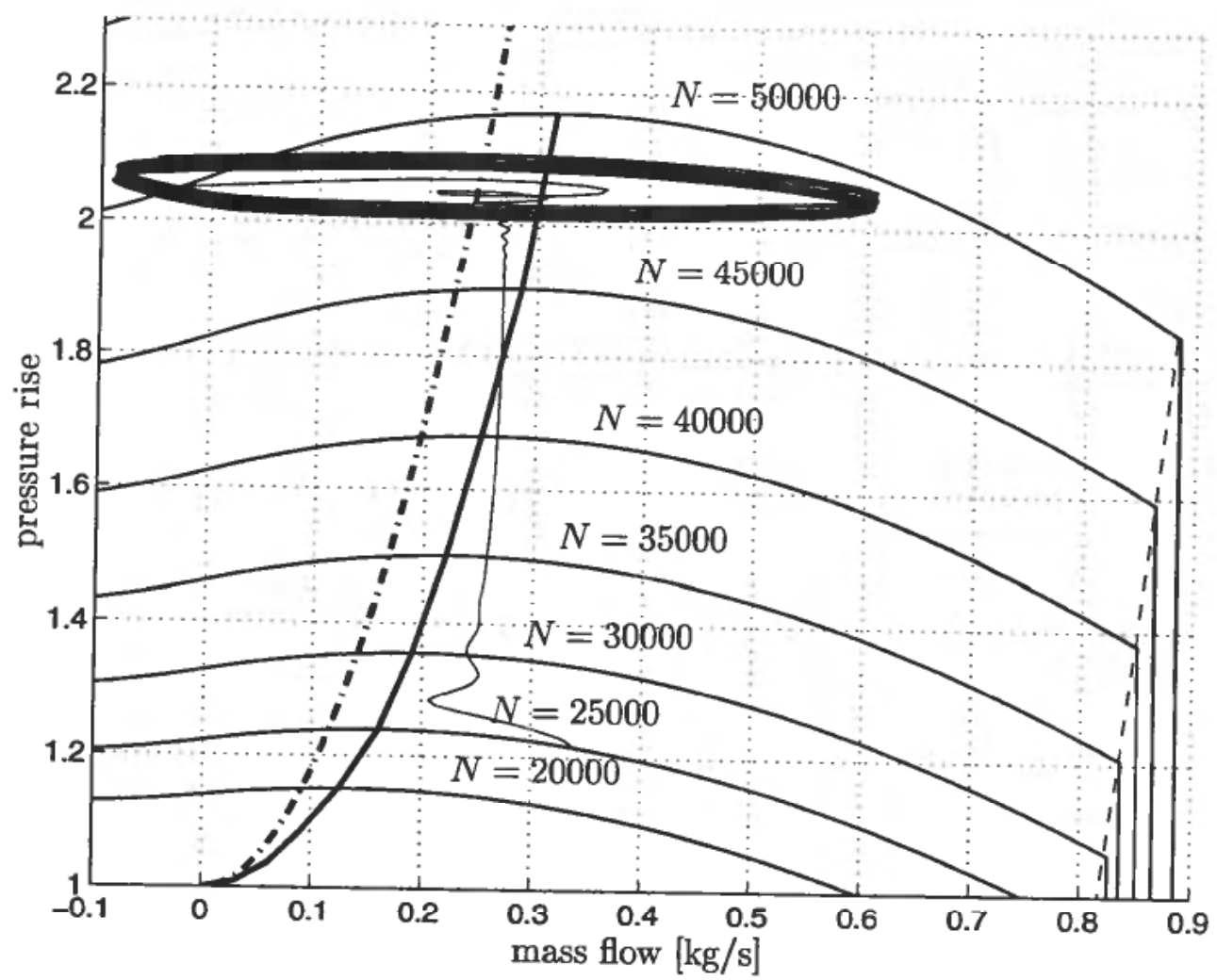

Figure 8. $\left(m(t), p(t) / p_{01}\right)$-trajectory plotted in the compressor characteristic. The throttle line is plotted with a dash-dot line, the surge line with a solid line and the stonewall with a dashed line. $N$ is the compressor speed in rpm. The surge cycle is clearly visible.

This simulation is also shown in Figure 8, where the pressure rise has been plotted versus the mass flow in the compressor characteristic. As can be seen, the compressor undergoes severe (deep) surge oscillations.

Now, the surge controller (38) is used with $k_{v}=0 \cdot 2$. The speed set point, speed controller parameters and throttle gain are as before. The results are shown in Figure 9. The desired speed is reached and the surge oscillations are eliminated. As previously mentioned there is a loss associated with the CCV control approach. The pressure drop over the valve is shown in the upper right corner of Figure 9. At equilibrium the pressure drop for this particular case is at ca. $5 \mathrm{kPa}$. Compared to the pressure rise over the compressor at this equilibrium, well over $200 \mathrm{kPa}$ this seems little when taken into account that the compressor now is operating in an area of the compressor map previously not possible. The CCV loss is dependent on the controller gain $k_{v}$. In this simulation the gain was set to $k_{v}=0.2$ to dominate the maximum positive slope of the compressor characteristic.

\section{Conclusion}

A surge control law and a PI speed control law for a centrifugal compression system have been developed. The modeling of the compressor characteristic was based on energy losses in the compressor stage. A close coupled valve was chosen as an actuator 

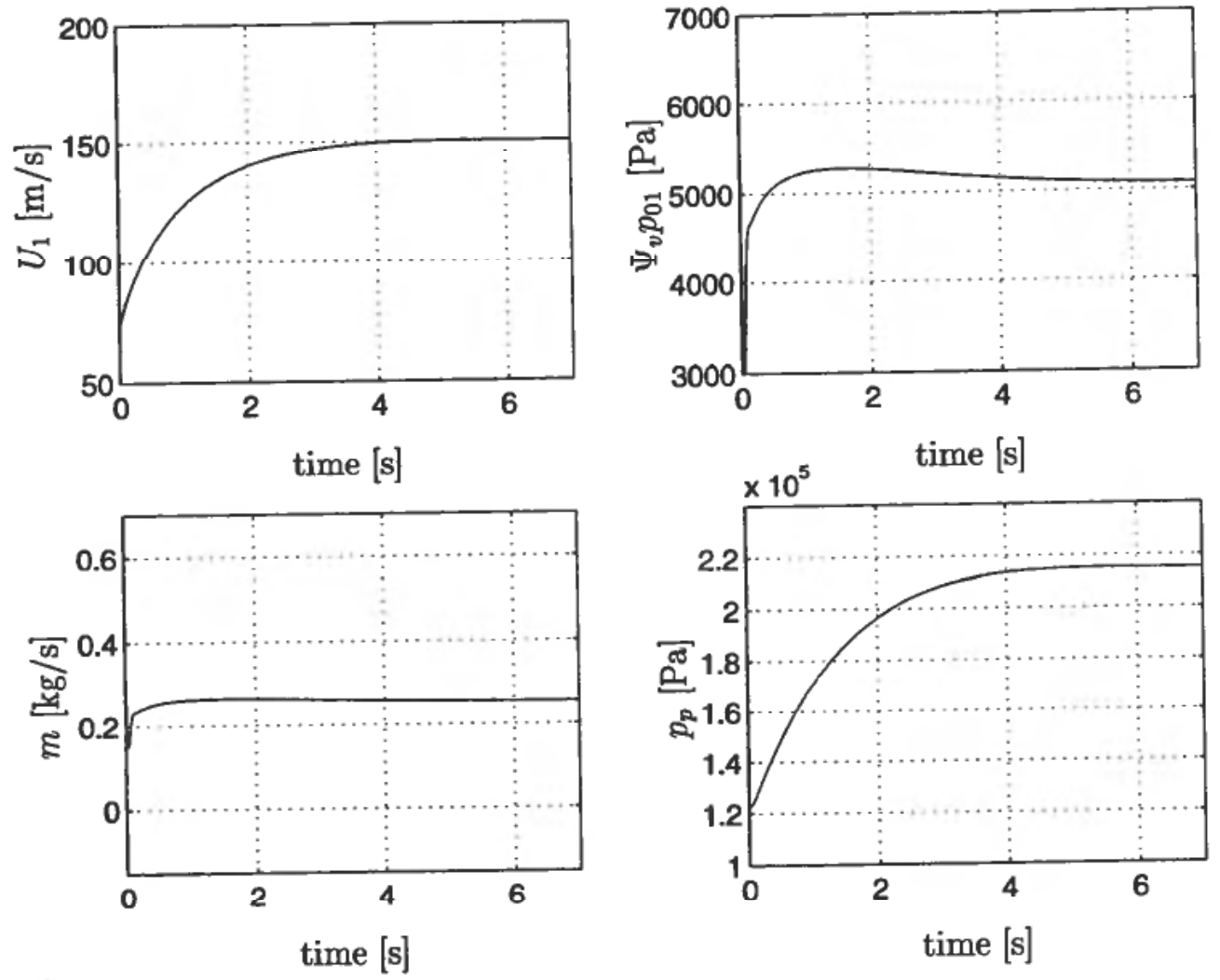

Figure 9. Same situation as in Figure 7, with surge control.

for the surge control. Using Lyapunovs method, the systems equilibrium was shown to be semi-global exponentially stable. Through simulations it was confirmed that the compressor can operate stable and reach desired speed in the previous unstable area to the right of the surge line in the compressor map.

REFERENCES

BALJÉ, O. E. (1952). A contribution to the problem of designing radial turbomachines. Transactions of the ASME, 74, 451-472.

CUMPSTY, N. A. (1989). Compressor Aerodynamics (Longman).

DE JAGER, B. (1995). Rotating stall and surge control: A survey. In Proceedings of the 35th Conference on Decision and Control. New Orleans, LA. pp. 1857-1862.

Dixon, S. L. (1978). Thermodynamics of turbomachinery, 3rd ed., Pergamon Press.

Dussourd, J. L., Pfannebecker, G. W. and Singhania, S. K. (1977). An experimental investigation of the control of surge in radial compressors using close coupled resistances. Journal of Fluids Engineering, 99, 64-76.

EVEKER, K. M. and NETT, C. N. (1991). Model development for active surge control rotating stall avoidance in aircraft gas turbine engines. In Proceedings of the 1991 American Control Conference. pp. 3166-3172.

FERGuSON, T. B. (1963). The centrifugal compressor stage. Butterworths, London.

FinK, D. A., Cumpsty, N. A. and GreitZer, E. M. (1992). Surge dynamics in a free-spool centrifugal compressor system. Journal of Turbomachinery, 114, 321-332.

GRAVDAHL, J.T. and EGELAND, O. (1997a). A Moore-Greitzer axial compressor model with spool dynamics. Accepted as a regular paper to the 36th IEEE Conference on Decision and Control.

GravdaHL, J. T. and EgELAND, O. (1997b). Compressor surge control using a close-coupled 
valve and backstepping. In Proceedings of the 1997 American Control Conference. Albuquerque, NM.

GRAVDAHL, J. T. and EgELAND, O. (1997c). Control of the three-state Moore-Greitzer compressor model using a close-coupled valve. In Proceedings of the 1997 European Control Conference.

Gravdahl, J. T. and Egeland, O. (1997d). Passivity based compressor surge control using a close-coupled valve. In A. Isidori and F. Allgöwer, editors, Proceedings of the 1997 COSY Workshop on Control of Nonlinear and Uncertain Systems. Zurich, Switzerland.
pp. 139-143.

GREITZER, E. M. (1976). Surge and Rotating stall in axial flow compressors. Part I: Theoretical compression system model. Journal of Engineering for Power, 98, 190-198.

GREITZER, E. M. (1981). The stability of pumping systems--The 1980 Freeman scolar lecture. Journal of Fluids Engineering, 103, 193-242.

Jungovski, W. M., WeISS, M. H. and PRICE, G. R. (1996). Pressure oscillations occuring in a centrifugal compressor system with and without passive and active surge control. Journal
of Turbomachinery, 118, 29-40.

HANSEN, K. E., JøRGENSEN, P. and LARSEN, P. S. (1981). Experimental and theoretical study of surge in a small centrifugal compressor. Journal of Fluids Engineering, 103, 391-394.

PinSLEY, J. E., GuenETTE, G. R., EpSTEIN, A. H. and GREITZER, E. M. (1991). Active stabilization of centrifugal compressor surge. Journal of Turbomachinery, 113, 723-732.

SimON, J. S. and VALAVANI, L. (1991). A Lyapunov based nonlinear control scheme for stabilizing a basic compression system using a close-coupled control valve. In Proceedings of the 1991 American Control Conference. pp. 2398-2406.

SimON, J. S., VALAVANi, L., EPSTEIN, A. H. and GREITZER, E. M. (1993). Evaluation of approaches to active compressor surge stabilization. Journal of Turbomachinery, 115, 57-67.

VAN DE WAL, M., WILLEMS, F. and DE JAGER, B. (1997). Selection of actuators and sensors for active surge control. Accepted as a regular paper to 1997 IEEE Conference on Control Applications.

WATSON, N. and JANOTA, M. S. (1982). Turbocharging the internal combustion engine (MacMillan).

WhitE, F. M. (1986). Fluid mechanics, 2nd ed. (McGraw-Hill, New York). 